\title{
Curcumin prevents tenofovir/lamivudine/efavirenz- induced nephrotoxicity in rats
}

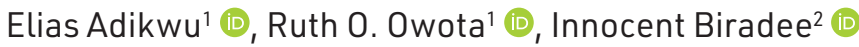 \\ 'Niger Delta University, Faculty of Pharmacy, Department of Pharmacology and Toxicology, Bayelsa State, Nigeria \\ ${ }^{2}$ University of Port Harcourt, School of Science Laboratory Technology, Department of Biomedical Technology, \\ Rivers State, Nigeria
}

ORCID IDs of the authors: E.A. 0000-0003-4349-8227; R.0.0. 0000-0003-3634-9856; I.B. 0000-0002-5377-0450

Cite this article as: Adikwu, E., Owota, R. O., \& Biradee, I. (2021). Curcumin prevents tenofovir/lamivudine/efavirenzinduced nephrotoxicity in rats. Istanbul Journal of Pharmacy, 51 (2), 221-227.

\begin{abstract}
Background and Aims: Nephrotoxicity is an adverse effect, which may occur with the use of tenofovir/lamivudine/efavirenz (TLE) in the treatment of human immunodeficiency virus (HIV) infection. Curcumin (CUM), an isolate of Curcuma longa L. is used in folk medicine for the treatment of ailments. This study attempts to establish whether CUM supplementation can protect against a rat model of TLE-induced nephrotoxicity.

Materials and Methods: Adult male Wistar rats $(n=40)$ were randomly grouped and supplemented orally with CUM $(50,100$ and $200 \mathrm{mg} / \mathrm{kg} / \mathrm{day})$ prior to the oral administration of TLE $(300 / 300 / 600 \mathrm{mg} / \mathrm{kg} /$ day) for 30 days. After the treatment, the rats were fasted overnight, weighed and anesthetized. Blood samples were collected, and sera were extracted for biochemical analyses. Kidney samples were excised, weighed and processed for oxidative stress markers and histology.

Results: Body weight was decreased $(p<0.01)$ whereas kidney weight was increased $(p<0.01)$ in TLE administered rats when compared to the control. Significant $(p<0.001)$ increments in serum uric acid, creatinine, urea and kidney malondialdehyde levels were observed in TLE-administered rats. Significant $(p<0.001)$ decreases in serum total protein, albumin, electrolytes, kidney superoxide dismutase, glutathione, catalase and glutathione peroxidase levels were observed in TLE administered rats when compared to the control. TLE produced tubular necrosis and hypercellular glomerulus with mesangial proliferation in the kidneys of treated rats. However, CUM $(50,100$ and $200 \mathrm{mg} / \mathrm{kg})$ supplementation abrogates TLE-induced nephrotoxicity in a dose-related manner at $p<0.05, p<0.01$ and $p<0.001$, respectively, when compared to TLE group.

Conclusion: CUM seems effective against TLE-induced nephrotoxicity.

Keywords: Antiretroviral, curcumin, nephrotoxicity, rat
\end{abstract}

\section{INTRODUCTION}

Highly active antiretroviral therapy (HAART) consists of three drugs active against human immununodeficiency virus (HIV) infection. HAART has significantly decreased HIV progression and prevents HIV-related infections. The success of HAART reflects on the reductions of $\mathrm{HIV}$ related morbidity and mortality in the world (Palella et al., 1998; Montaner et al., 2010). However, the use of HAART has been associated with a myriad of toxicities, especially nephrotoxicity. Acute kidney injury, tubulopathies, chronic kidney disease, and end-stage renal disease requiring renal replacement therapy have been documented with the use of HAART (Kalyesubula \& Perazella, 2016).

Tenofovir -lamivudine -efavirenz (TLE) is an integral part of the preferred first-line regimens for the treatment of HIV in adolescents and antiretroviral-naive adults especially in resource-limited settings (WHO, 2013). It has reduced the incidence of HIV related death, but the incidence of nephrotoxicity attributed to its tenofovir component, which can be aggravated by partner drugs is a worrisome challenge. An incidence of $17-22 \%$ of tubular dysfunction was observed in tenofovir containing regi- 
mens. Also, an incidence of $18.3 \%$ moderate renal impairment and $2.3 \%$ severe renal impairment were documented with the use of tenofovir containing regimens (Nartey et al., 2019). The primary clinical presentations of nephrotoxicity caused by tenofovir include proximal tubular dysfunction, electrolytes and acid-base disorder (Perazella, 2010). Kidney histological aberrations include chronic tubular-interstitial scarring, necrosis, tubular atrophy and interstitial fibrosis (Herlitz et al., 2010).

Curcumin (diferuloyl methane) is a low-molecular weight compound extracted from the roots of Curcuma longa L. (Zingiberaceae). It is traditionally used for centuries in Asia and other parts of the world for medicinal and culinary purposes. CUM has a diverse and wide range of targets at molecular and cellular levels (Noorafshan \& Ashkani-Esfahani, 2013). Substantial number of in-vitro and in-vivo studies showed that it has essential pharmacological activities including anti-inflammatory, antispasmodic, antioxidant, anti-cancer, and antimicrobial effects (Akram et al., 2010). In folk medicine, it is used as treatments for sprains, liver disorders, anorexia, rheumatism, diabetes, cough, sinusitis and inflammation (Noorafshan \& Ashkani-Esfahani, 2013). It has redox regulatory effects such as scavenging of free radicals and increased antioxidant activities (Hewlings \& Kalman, 2007). Its anti-inflammatory activity has been characterized by reduction in pro-inflammatory cytokines including tumor necrosis factor alpha (TNF-a), interleukin 6 (IL-6) and the inhibition of NF-kappa B pathway (Noorafshan \& Ashkani-Esfahani, 2013). CUM has been shown to have potential protective effect on experimentally induced toxicities including hepatotoxicity (Farghaly \& Hussein, 2010) cardiotoxicity (Mohantya et al., 2004) and nephrotoxicity (El-Zawahry \& Abu El Kheir, 2007). This study examined its protective activity against TLE-induced nephrotoxicity in Wistar rats, which is a novel study.

\section{MATERIALS AND METHODS}

\section{Animals, drugs, chemicals and treatment}

Tenofovir disoproxil fumarate/lamivudine/ efavirenz (TLE), Curcuma longa (CUM), and piperine were used.

Adult male Wistar rats $(n=40)$ were randomly grouped into 8 of $n=5 /$ group and used. The rats were acclimated for 2 weeks in cages in a standardized condition (12 h light/day cycles, $25^{\circ} \mathrm{C} \pm 5^{\circ} \mathrm{C}$ ) with ad libitum access to food and water. The rats were purchased from the animal research unit of the Department of Pharmacology, Faculty of Basic Clinical Sciences, University of Port Harcourt, Rivers State, Nigeria. The guideline (2020/569/EU) on animal handling prepared by European Parliament and of the Council was used for this study. Ethical approval for this study (NDU/PHARM/PCO/AEC/064B) was provided by the Research Ethics Committee of the Department of Pharmacology/Toxicology, Faculty of Pharmacy, Niger Delta University on 4 August 2020.

\section{Animal treatment}

Group 1 (Control) received normal saline (0.2 mL/day/p.o). Groups 2-4 received CUM (50, 100 and 200 mg/kg/day/p.o) (Lee et al., 2016). Group 5 received TLE (300/300/600 mg/kg/ day/p.o). Groups 6-8 received CUM (50, 100 and 200 mg/kg/ day/p.o) before receiving TLE (300/300/600 mg/kg/day/p.o).
Piperine (20 mg/kg/p.o) was added to CUM to improve bioavailability (Shoba et al., 1998). All the rats were treated for 30 days. After the treatment, the rats were allowed to fast overnight, weighed and anesthetized through inhalation in a chamber of diethyl ether. Blood samples were collected from the heart, centrifuged (1500 rpm for $20 \mathrm{~min}$ ) and sera were extracted for biochemical assessments. Kidney samples were collected, rinsed in cold saline and homogenized in 0.1 M Tris- $\mathrm{HCl}$ solution buffered ( $\mathrm{pH}$ 7.4). The homogenates were centrifuged (3000 rpm for 15 min), supernatants decanted and assayed for oxidative stress markers.

\section{Assessment of serum biochemical markers}

Serum total protein, creatinine, albumin, uric acid, urea, sodium, bicarbonate potassium, and chloride and concentrations were measured using laboratory test kits.

\section{Assessment of kidney oxidative stress markers}

Malondialdehyde (MDA) was assayed as reported by Buege \& Aust, 1978. Reduced glutathione (GSH) was assayed using the method reported by Sedlak \& Lindsay, 1968. Catalase (CAT) was assayed as described by Aebi, 1984. Glutathione peroxidase (GPx) was measured according to Rotruck et al., 1973. Superoxide dismutase (SOD) was assessed as reported by Sun \& Zigman, 1978.

\section{Histological analysis}

Kidney samples were collected, blotted and fixed in 10\% buffered formaldehyde. Kidney samples were processed and embedded in paraffin. Sections ( $3 \mu \mathrm{m}$ in thickness) were produced on slides and stained with hematoxylin and eosin (H\&E). Stained sections were assessed for histological changes using a light microscope.

\section{Statistical analysis}

Mean \pm standard error of mean (SEM) for the results for all groups ( $n=5$ ) was determined. Data was analyzed using oneway analysis of variance (ANOVA) followed by Tukey's posthoc test. Graph Pad Prism 5 Software (San Diego, CA USA) was used for data analysis. Significance was set at $p<0.05, p<0.01$ and $\mathrm{p}<0.001$.

\section{RESULTS}

\section{Effects of curcumin on body and kidney weights of teno- fovir/lamivudine/efavirenz -treated rats}

The effects of CUM on body and kidney weights were not significant ( $p>0.05$ ) when compared to control (Table 1). The administration of TLE produced a significant $(p<0.01)$ decrease in body weight with a significant increase in kidney weight $(p<0.01)$ when compared to control (Table 1). However, body and kidney weights were restored in $\operatorname{CUM}(50,100$, and 200 $\mathrm{mg} / \mathrm{kg}$ ) supplemented rats at $p<0.05, p<0.01$, and $p<0.01$, respectively when compared to TLE group (Table 1).

\section{Effect of curcumin on serum kidney function markers of tenofovir/lamivudine/efavirenz -treated rats}

The administration of CUM had no significant ( $p>0.05$ ) effects on serum total protein, potassium, chloride, sodium, bicarbonate, albumin, uric acid, creatinine and urea levels when 
compared to control (Table 2) (Figures 1-5). In TLE administered rats, serum total protein, potassium, chloride, sodium, bicarbonate and albumin levels were decreased significantly $(p<0.001)$ whereas uric acid, creatinine and urea levels were increased significantly $(p<0.001)$ when compared to control (Table 2) (Figures 1-5). However, CUM (50, 100, and 200 mg/kg)

Table 1. Effects of curcumin on body and kidney weights of tenofovir/lamivudine/efavirenz -treated rats.

\begin{tabular}{|c|c|c|c|}
\hline Dose (mg/kg) & FBW (g) & $A K W(g)$ & RKW (\%) \\
\hline Control & $255.8 \pm 17.7$ & $0.65 \pm 0.06$ & $0.25 \pm 0.06$ \\
\hline CUM 50 & $250.1 \pm 15.1$ & $0.67 \pm 0.01$ & $0.27 \pm 0.03$ \\
\hline CUM 100 & $252.7 \pm 13.0$ & $0.63 \pm 0.09$ & $0.25 \pm 0.01$ \\
\hline CUM 200 & $250.2 \pm 15.9$ & $0.66 \pm 0.04$ & $0.26 \pm 0.06$ \\
\hline TLE & $161.5 \pm 17.6 \#$ & $1.99 \pm 0.03 \#$ & $1.23 \pm 0.09 \#$ \\
\hline CUM 50 + TLE & $180.3 \pm 16.6$ & $1.70 \pm 0.02$ & $0.94 \pm 0.07$ * \\
\hline CUM $100+$ TLE & $200.7 \pm 16.1^{*}$ & $1.16 \pm 0.06$ * & $0.58 \pm 0.05^{\star \star}$ \\
\hline CUM $200+$ TLE & $250.8 \pm 18.6$ ** & $0.70 \pm 0.08 * *$ & $0.28 \pm 0.01 * * *$ \\
\hline
\end{tabular}

Table 2. Effect of curcumin on serum electrolytes of tenofovir/lamivudine/efavirenz-treated rats.

\begin{tabular}{lcccc}
$\begin{array}{l}\text { Dose } \\
(\mathrm{mg} / \mathrm{kg})\end{array}$ & $\begin{array}{c}\text { Potassium } \\
(\mathrm{mmo} / \mathrm{L})\end{array}$ & $\begin{array}{c}\text { Chloride } \\
(\mathrm{mmo} / \mathrm{L})\end{array}$ & $\begin{array}{c}\text { Sodium } \\
(\mathrm{mmo} / \mathrm{L})\end{array}$ & Bicarbonate $(\mathrm{mmo} / \mathrm{L})$ \\
\hline Control & $3.70 \pm 0.19$ & $111.02 \pm 12.0$ & $125.63 \pm 11.3$ & $12.86 \pm 1.45$ \\
CUM 50 & $3.71 \pm 0.04$ & $112.11 \pm 13.9$ & $126.16 \pm 12.0$ & $12.67 \pm 2.17$ \\
CUM 100 & $3.73 \pm 0.30$ & $114.12 \pm 10.7$ & $128.87 \pm 13.2$ & $12.52 \pm 3.63$ \\
CUM 200 & $3.78 \pm 0.06$ & $116.76 \pm 12.8$ & $129.74 \pm 14.8$ & $12.35 \pm 2.33$ \\
TLE & $1.57 \pm 0.17^{\pi}$ & $48.10 \pm 5.57^{\mathrm{r}}$ & $48.03 \pm 4.66^{\pi}$ & $5.00 \pm 0.76^{\pi}$ \\
CUM 50+TLE & $2.18 \pm 0.06^{\mathrm{a}}$ & $65.02 \pm 6.86^{\mathrm{a}}$ & $67.16 \pm 6.92^{\mathrm{a}}$ & $7.02 \pm 0.09^{\mathrm{a}}$ \\
CUM 100+TLE & $2.89 \pm 0.43^{\mathrm{b}}$ & $85.07 \pm 8.66^{\mathrm{b}}$ & $88.34 \pm 8.55^{\mathrm{b}}$ & $9.00 \pm 1.22^{\mathrm{b}}$ \\
CUM 200+TLE & $3.60 \pm 0.36^{\mathrm{c}}$ & $109.11 \pm 10.7^{\mathrm{c}}$ & $118.78 \pm 11.1^{\mathrm{c}}$ & $12.48 \pm 1.60^{\mathrm{c}}$
\end{tabular}

CUM: Curcumin, TLE: Tenofovir/lamivudine/efavirenz, $n=5$, Data as mean \pm SEM (Standard error of mean), ${ }^{n} p<0.001$ Significant differnce when compared to control, a $p<0.05,{ }^{b} p<0.01,{ }^{c} p<0.001$ Significant differnce when compared to TLE.

Table 3. Effect of curcumin on kidney oxidative stress markers of tenofovir/lamivudine/efavirenz-treated rats.

\begin{tabular}{|c|c|c|c|c|c|}
\hline $\begin{array}{l}\text { Dose } \\
\text { (mg/kg) }\end{array}$ & $\begin{array}{l}\text { MDA (mmol/mg } \\
\text { protein) }\end{array}$ & $\begin{array}{l}\text { GSH ( } \mu \text { mole/ } \\
\text { mgprotein) }\end{array}$ & $\begin{array}{c}\text { CAT } \\
\text { (U/mgprotein) }\end{array}$ & $\begin{array}{c}\text { SOD (U/ } \\
\text { mgprotein) }\end{array}$ & $\begin{array}{c}\text { GPx (U/ } \\
\text { mgprotein) }\end{array}$ \\
\hline Control & $0.17 \pm 0.06$ & $20.03 \pm 3.89$ & $29.55 \pm 3.24$ & $23.05 \pm 3.77$ & $30.34 \pm 4.87$ \\
\hline CUM 50 & $0.16 \pm 0.09$ & $20.27 \pm 2.45$ & $29.73 \pm 2.56$ & $23.37 \pm 2.98$ & $30.51 \pm 3.96$ \\
\hline CUM 100 & $0.15 \pm 0.05$ & $20.61 \pm 2.40$ & $30.04 \pm 3.01$ & $23.54 \pm 4.70$ & $30.70 \pm 4.33$ \\
\hline CUM 200 & $0.13 \pm 0.07$ & $20.90 \pm 3.73$ & $30.53 \pm 4.66$ & $24.06 \pm 3.66$ & $31.05 \pm 4.16$ \\
\hline TLE & $2.51 \pm 0.73^{\pi}$ & $4.66 \pm 0.73^{\pi}$ & $7.14 \pm 0.67^{\pi}$ & $7.36 \pm 0.72^{\pi}$ & $8.05 \pm 0.56^{\pi}$ \\
\hline CUM 50+TLE & $1.80 \pm 0.81^{\mathrm{a}}$ & $7.54 \pm 0.84^{\mathrm{a}}$ & $10.27 \pm 1.09^{a}$ & $11.47 \pm 1.89^{a}$ & $11.67 \pm 1.22^{\mathrm{a}}$ \\
\hline CUM 100+TLE & $0.94 \pm 0.06^{b}$ & $11.64 \pm 1.02^{\mathrm{b}}$ & $16.65 \pm 1.67^{b}$ & $16.50 \pm 1.89^{b}$ & $17.83 \pm 1.01^{b}$ \\
\hline CUM 200+TLE & $0.20 \pm 0.08^{c}$ & $18.92 \pm 2.16^{c}$ & $26.92 \pm 4.27^{c}$ & $21.73 \pm 2.02^{c}$ & $27.42 \pm 3.50^{c}$ \\
\hline
\end{tabular}




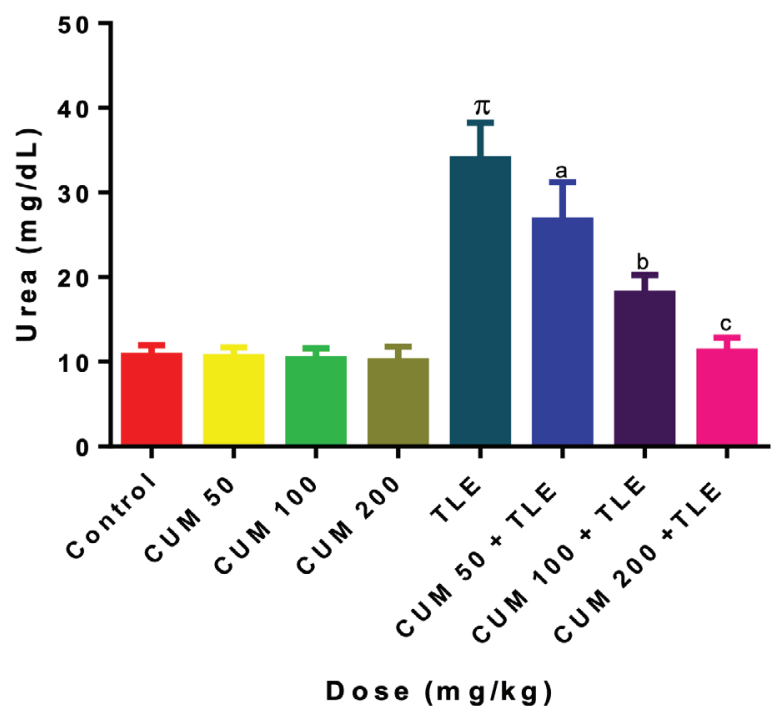

Figure 1. Effect of curcumin on serum urea levels of tenofovir/lamivudine/efavirenz -treated rats.

CUM: Curcumin, TLE: Tenofovir/lamivudine/efavirenz, Data as mean \pm SEM (Standard error of mean), $n=5,{ }^{n} p<0.001$ significance when compared to control, ${ }^{a} p<0.05,{ }^{b} p<0.01,{ }^{c} p<0.001$ significance when compared to TLE.

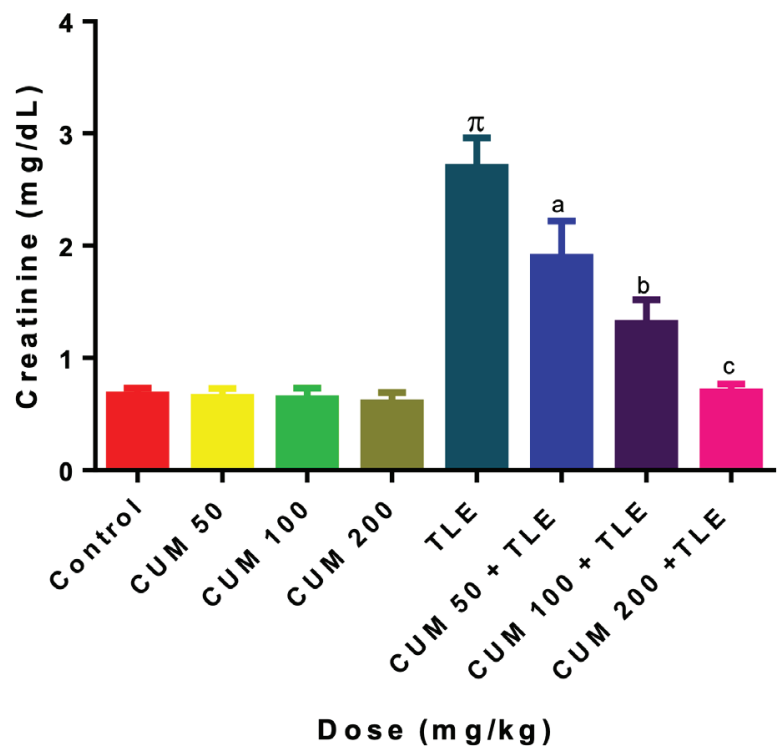

Figure 2. Effect of curcumin on serum creatinine levels of tenofovir/ lamivudine/efavirenz -treated rats.

CUM: Curcumin, TLE: Tenofovir/lamivudine/efavirenz, Data as mean \pm SEM (Standard error of mean), $n=5,{ }^{\pi} p<0.001$ significant difference when compared to control, ${ }^{a} p<0.05,{ }^{b} p<0.01,{ }^{c} p<0.001$ significant difference when compared to TLE.

supplementation increased serum total protein, potassium, chloride, sodium, bicarbonate and albumin levels significantly at $p<0.05, p<0.01$, and $p<0.01$, respectively when compared to TLE. CUM (50, 100, and $200 \mathrm{mg} / \mathrm{kg}$ ) supplementation decreased serum uric acid, creatinine and urea levels in a doserelated manner at $p<0.05, p<0.01$, and $p<0.001$, respectively

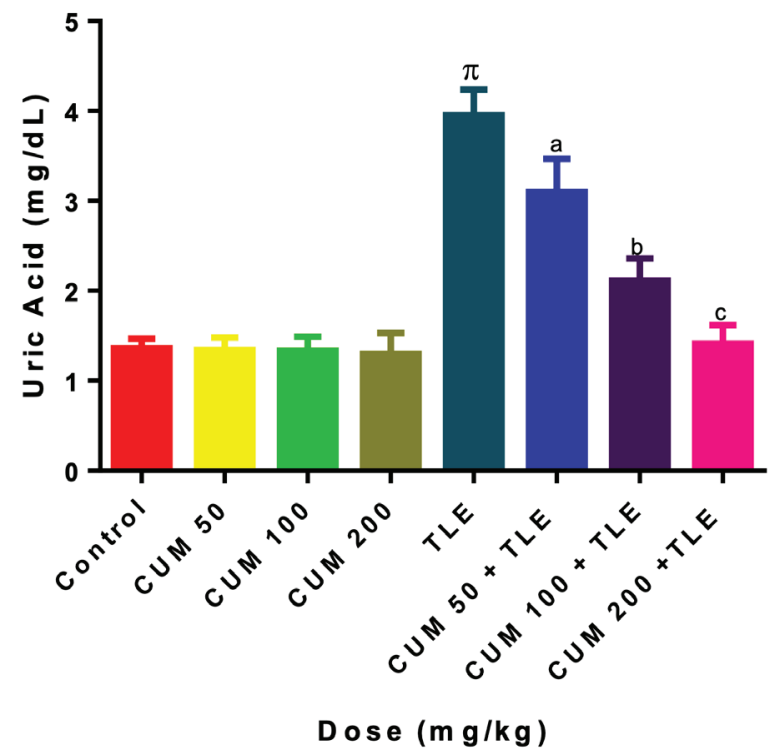

Figure 3. Effect of curcumin on serum uric acid levels of tenofovir/ lamivudine/efavirenz -treated rats.

CUM: Curcumin, TLE: Tenofovir/lamivudine/efavirenz, Data as mean \pm SEM (Standard error of mean), $n=5,{ }^{\pi} p<0.001$ significant difference when compared to control, ${ }^{a} p<0.05,{ }^{b} p<0.01,{ }^{c} p<0.001$ significant difference when compared to TLE.

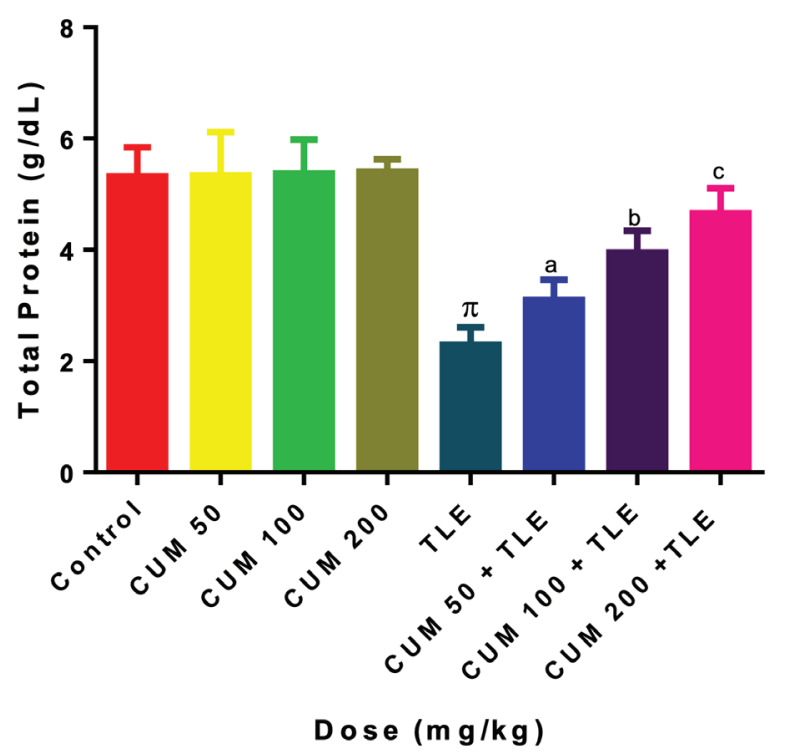

Figure 4. Effect of curcumin on serum total protein levels of tenofovir/ lamivudine/efavirenz -treated rats.

CUM: Curcumin, TLE: Tenofovir/lamivudine/efavirenz, Data as mean \pm SEM (Standard error of mean), $n=5, \pi p<0.001$ significant difference when compared to control, ${ }^{a} p<0.05,{ }^{b} p<0.01,{ }^{c} p<0.001$ significant difference when compared to TLE.

\section{Effects of curcumin on serum kidney oxidative markers} and histology of tenofovir/lamivudine/efavirenz -treated rats

Kidney antioxidants (SOD, CAT, GSH and GPX) and MDA levels were normal $(p>0.05)$ in CUM-administered rats when compared to control. TLE administration significantly $(p<0.001)$ 


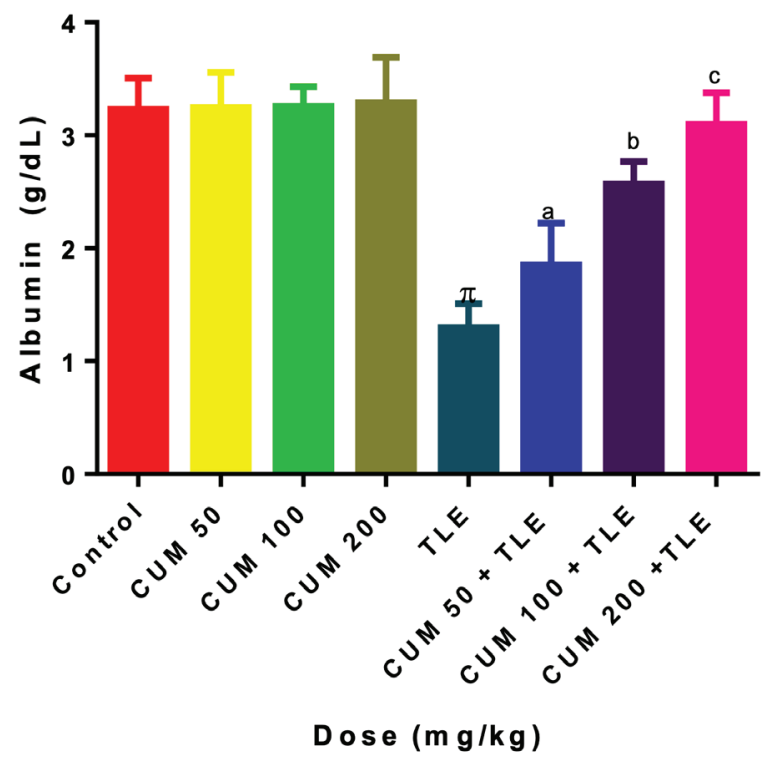

Figure 5. Effect of curcuminon serum albumin of tenofovir/lamivudine/efavirenz-treated rats.

CUM: Curcumin, TLE: Tenofovir/lamivudine/efavirenz, Data as mean \pm SEM (Standard error of mean), $n=5, \pi p<0.001$ significant difference when compared to control, ${ }^{a} p<0.05,{ }^{b} p<0.01,{ }^{c} p<0.001$ significant difference when compared to TLE.

decreased kidney antioxidants, but significantly $(p<0.001)$ increased kidney MDA levels when compared to control (Table 3). However, CUM (50, 100, and $200 \mathrm{mg} / \mathrm{kg}$ ) supplementation significantly increased kidney antioxidants, and significantly decreased kidney MDA levels in a dose-related manner at $p<0.05, p<0.01$, and $p<0.001$, respectively when compared to TLE treated rats (Table 3). The kidney of the control rats showed normal renal tubule and glomerulus (Fig 6A), but the kidney of TLE-treated rat showed tubular necrosis and hypercellular glomerulus with mesangial proliferation (Figure 6B). The kidney of CUM $(25 \mathrm{mg} / \mathrm{kg})$ and CUM $(50 \mathrm{mg} / \mathrm{kg})$ supplemented rats showed normal renal tubules and hypercellular glomeruli with mesangial proliferations as shown in (Figure 6C) and (Figure 6D), respectively. However, the kidney of CUM $(100 \mathrm{mg} / \mathrm{kg})$ supplemented rat showed normal renal tubules and glomerulus (Figure 6E).

\section{DISCUSSION}

TLE-related nephrotoxicity can add to HIV infection associated socio-economic burden (Perazella, 2010). CUM is an isolate of turmeric with a wide spectrum of biological activity that is used in folk medicine (Noorafshan \& Ashkani-Esfahani, 2013). This study attempts to establish whether CUM supplementation can prevent TLE-induced nephrotoxicity in a rat model. In the current study, the administration of CUM had no effects on all evaluated parameters at the serum and tissue levels. On the other hand, the administration of TLE increased kidney weight, but decreased body weight, which supports earlier reports (Jang et al., 2010). The observed decrease in body weight may be ascribed to decreased appetite whereas increase in kidney weight may be predicated on the induc- tion of inflammation by TLE. In this study, the conspicuous incapacitation of kidney function by TLE was marked by elevated serum uric acid, urea, and creatinine levels with decreased serum total protein, albumin and serum electrolytes. This observation supports previous reports (Fenandez-Fernandez et al., 2011). The health status of the kidney is ascribed to its capacity to functionally regulate the serum concentrations of the aforementioned indices (Gowda et al., 2010). In the midst of perturbations caused by chemical assaults or diseases the functional capacity of the kidney is impaired causing aberrations in serum uric acid, urea, creatinine, total protein, albumin and serum electrolytes (Gowda et al., 2010). In the present study, TLE caused dysfunction in kidney reduction/ oxidation status of treated rats characterized by decreased antioxidants and increased MDA levels. This observation supports earlier findings (Adikwu \& Apiakise, 2016). Antioxidants form defensive network that prevents oxidative damage by scavenging and neutralizing free radicals, but could be consumed and depleted as a consequence of increased free radicals production beyond antioxidants regulation causing oxidative stress (Adikwu \& Apiakise, 2016). Hence, depleted kidney antioxidants observed in TLE-treated rats established oxidative stress. MDA is used experimentally to mirror the occurrence of lipid peroxidation (LPO) in a pathologic process or condition (Adikwu \& Apiakise, 2016). Therefore, TLEinduced elevation in MDA level established the occurrence of LPO. In the current study, TLE-induced nephrotoxicity was characterized by kidney tubular necrosis and hypercellular glomerulus with mesangial proliferation. This observation is in agreement with earlier findings (Herlitz et al., 2010). In the current study, CUM supplementation abrogates TLE-induced nephrotoxicity in a dose-related manner. This was characterized by restored body and kidney weights and up-regulation of serum total protein, albumin, electrolytes and kidney antioxidants. CUM supplementation caused down-regulation of serum uric acid, creatinine, urea and kidney MDA levels. Also, tubular necrosis and hypercellular glomerulus with mesangial proliferation were absent in the kidneys of rats supplemented with the highest dose of CUM. This finding correlates with the reported protective activity of CUM against gentamicin-induced nephrotoxicity in rats (El-Zawahry \& Abu El Kheir, 2007). In the present study, the protective impact of CUM on TLE-induced nephrotoxicity may be ascribed to its antioxidant and anti-inflammatory activities. Studies have associated most therapeutic effects of CUM to its antioxidant and anti-inflammatory activities (Hewlings \& Kalman, 2007). CUM, as an antioxidant, inhibits oxidative stress and LPO by scavenging and neutralizing ROS (Menon \&Sudheer, 2007). It can up regulate the activities of endogenous antioxidants (SOD, CAT, and GSH) and increase antioxidants gene expression. CUM can inhibit enzymes including xanthine hydrogenase/oxidase and lipoxygenase/cyclooxygenase, which are facilitators of free radicals production and can decrease the gene expression of such enzymes (Lin et al., 2007). The antiinflammatory action of CUM includes inhibitory effects on pro-inflammatory mediators such as cytokines (TNF- $a$ and IL6). It can also inhibits NF-kappaB activation pathway, which is essential for inflammation (Noorafshan \& Ashkani-Esfahani, 2013; Lin et al., 2007). 

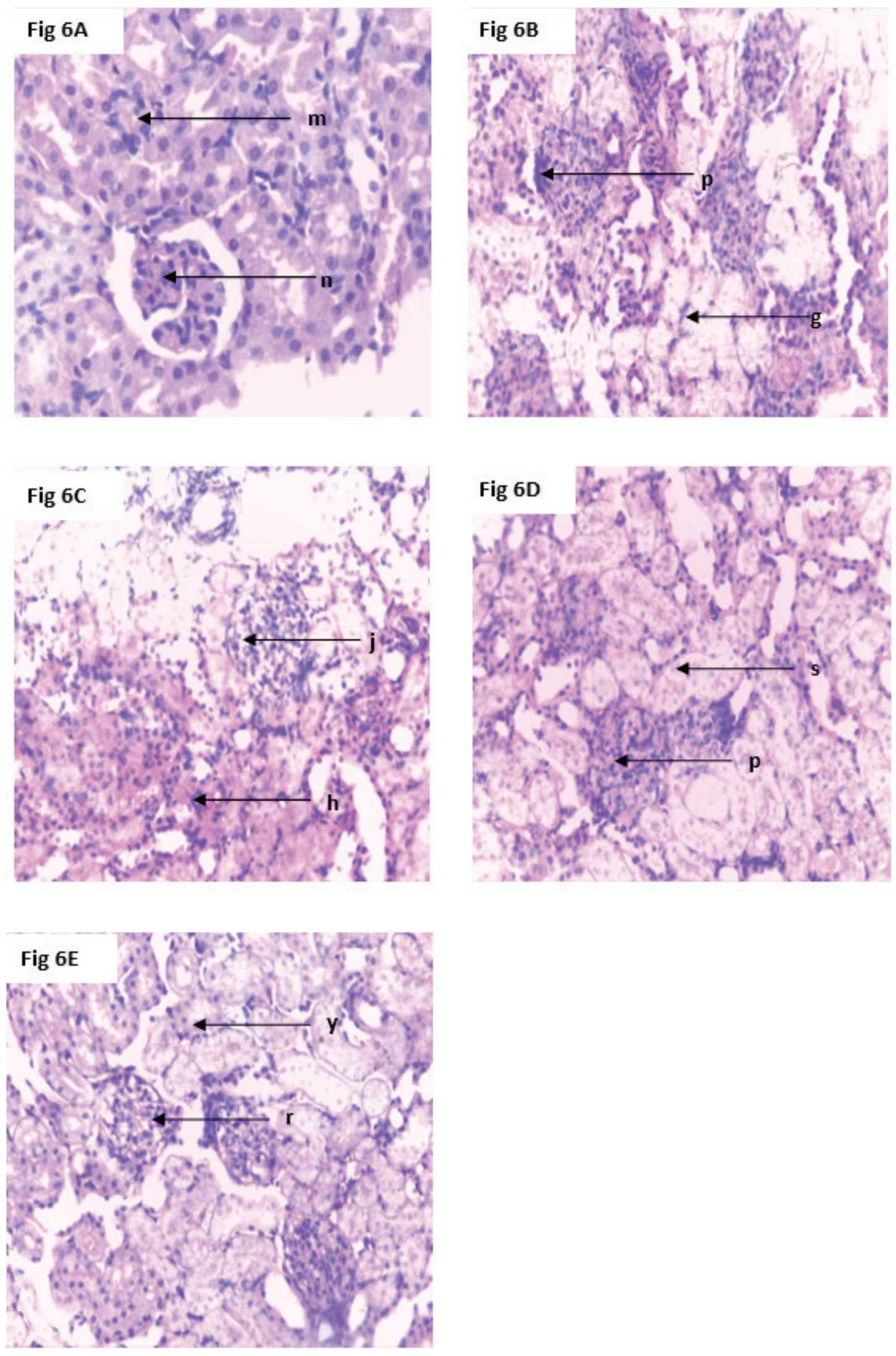

Figure 6. A: Kidney of control rat showed normal renal tubule ( $\mathrm{n}$ ) and glomerulus (m); B: Kidney of TLE-treated rat showed tubular necrosis (h) and hypercellular glomerulus with mesangial proliferation (j); C: Kidney of CUM ( $25 \mathrm{mg} / \mathrm{kg}$ ) supplemented rat showed normal renal tubule (h) and hypercellular glomerulus with mesangial proliferation (j); D: Kidney of CUM (50mg/kg) supplemented rat showed normal renal tubule (s) and hypercellular glomerulus with mesangial proliferation (p); E: Kidney of CUM (100mg/kg) supplemented rat showed normal renal tubule (y) and glomerulus ( $r$. 


\section{CONCLUSION}

Based on the observation in the current study, CUM may clinically protect against TLE-related nephrotoxicity.

Peer-review: Externally peer-reviewed.

Author Contributions: Conception/Design of Study- E.A., R.O.O., I.B.; Data Acquisition- E.A., R.O.O., I.B.; Data Analysis/Interpretation- E.A., R.O.O., I.B.; Drafting Manuscript- E.A., R.O.O., I.B.; Critical Revision of Manuscript- E.A., R.O.O., I.B.; Final Approval and Accountability- E.A., R.O.O., I.B.

Conflict of Interest: The authors have no conflict of interest to declare.

Financial Disclosure: Authors declared no financial support.

Acknowledgement: The authors appreciate Mr Cosmos Obi, Department of Pharmacology/ Toxicology, Faculty of Pharmacy, Niger Delta University, Bayelsa State, Nigeria.

\section{REFERENCES}

- $\quad$ Adikwu., E., \& Apiakise W. (2016). Ameliorative effects of vitamins $\mathrm{c}$ and e on tenofovir/nevirapine-induced hepatorenal oxidative stress in albino rats. Indonesian Journal ofPharmacy, 27, 211-219.

- $\quad$ Aebi, H. (1974). Catalase in vitro. In S.P. Colowick \& N. O. Kaplane (Eds.), Method in Enzymology (pp. 673-685). New York, USA: Academic Press.

- Akram, M., Uddin, S., Ahmed, A., Usmanghani, K., Hannan, A., Mohiuddi, E., \& Asif, M. (2010). Curcuma longa and Curcumin: A Review Article. Romania Journal of Biology Plant Biology, 55, 65-70.

- Buege, J. A., \& Aust, S. D. (1978). Microsomal lipid peroxidation. Methods in Enzymology, 52, 302-310. https://doi.org/10.1016/ S0076-6879(78)52032-6.

- El-Zawahry, B. H., \& Abu El Kheir E. M. (2007). The min against gentamicin-induced renal dysfunction and Oxidative Stress in male albino rats. The Egyptian Journal of Hospital Medicine, 29, 546-556. https://doi.org/10.12816/EJHM.2007.17699

- Farghaly, H. S., \& Hussein, M. A. (2010). Protective effect of curcumin against paracetamol-induced liver damage. AustralianJournal of Basic and Applied Sciences, 4(9), 4266-4274.

- Fernandez-Fernandez, B., Montoya-Ferrer, A., Sanz, A. B., SanchezNiño, M., Izquierdo, M. C., Poveda, J., \& Aberto, O. (2011). Tenofovir Nephrotoxicity: 2011 Update. AIDS Research and Treatment, 2011, 1-11. https://doi.org/10.1155/2011/354908

- Gowda, S., Desai, P. B., Kulkarni, S. S., Hull, V. V., Math, A. A., \& Vernekar, S. N. (2010). Markers of renal function tests. North American Journal of Medical Sciences, 2(4), 170-173.

- Herlitz, L.C., Mohan, S., Stokes, M. B., Radhakrishnan, J., D'Agati, V. D., \& Markowitz, G.S. (2010). Tenofovir nephrotoxicity: Acute tubular necrosis with distinctive clinical, pathological, and mitochondrial abnormalities. Kidney International, 78(11), 1171-1177. https://doi.org/10.1038/ki.2010.318

- Hewlings, J. S., \& Kalman, D. S. (2007). Curcumin. A Review of Its'Effects on Human Health. Foods, 92, 2-11. https://doi.org/10.3390/ foods6100092

- Jang, E., Lee, J. K., Inn, K., Chung, E. K., Lee, K., \& Lee, J. (2010). Renal Dysfunction and Tubulopathy Induced by High-Dose Tenofovir Disoproxil Fumarate in C57BL/6 Mice, Healthcare, 8, 417, 2-10. https://doi.org/10.3390/healthcare8040417

- Kalyesubula, R., \& Perazella, M. A. (2016). Nephrotoxicity of HAART. AIDS Research and Treatment 2016, 1-11 https://doi. org/10.1155/2011/562790
- Lee, H., Kim, S., Lee G., Choi, M., Jung, H., Kim Y. J., Kwon, H. \& Chae, H. (2016). Turmeric extract and its active compound, curcumin, protect against chronic CCl4-induced liver damage by enhancing antioxidation. BMC Complementary and Alternative Medicine, 16, 1-9. https://doi.org/10.1186/s12906-016-1307-6

- $\quad$ Lin, Y. G., Kunnumakkara, A. B., Nair, A., Merritt, W. M., Han, L. Y., Armaiz-Pena, G. N. ... Sood, A. K. (2007). Curcumin inhibits tumor growth and angiogenesis in ovarian carcinoma by targeting the nuclear factor-KB pathway. Clinical Cancer Research, 13, 34233430. https://doi.org/10.1158/1078-0432.CCR-06-3072

- Menon, V. P., \& Sudheer, A. R. (2007). Antioxidant and anti-inflammatory properties of curcumin. Advances in Experimental Medicine and Bioliogy, 595, 105-125. https://doi.org/10.1007/978-0387-46401-5_3

- Mohantya, I., Singh, A. D., Amit, D., Joshia. S., Keval, K.T., \& Gupta, S. K., (2004). Protective effects of Curcuma longa on ischemia-reperfusion induced myocardial injuries and their mechanisms. Life Sciences, 75, 1701-1709. https://doi.org/10.1016/j.lfs.2004.02.032.

- Montaner, J. S. G., Wood, E., Kerr, T., Lima. V., Barrios, R., Shannon, K, ......Hogg R. (2010). Expanded highly active antiretroviral therapy coverage among HIV-positive drug users to improve individual and public health outcomes. Journal of Acquired Immune Deficiency Syndromes, 55(1), 5-9. https://doi.org/10.1097/ QAl.0b013e3181f9c1f0

- $\quad$ Nartey, E. T., Tetteh, R. A., Yankey, B. A., Nartey E. T., Lartey M., Leukens H. G. M., \& Dodoo A. N. (2019). Tenofovir-associated renal toxicity in a cohort of HIV infected patients in Ghana. BMC Research Notes, 12, 2-6. https://doi.org/10.1186/s13104-019-4454-2 Noorafshan, A., \& Ashkani-Esfahani, S. (2013). Review of therapeutic effects of curcumin. Current Pharmaceutical Design, 19, 20322046. https://doi.org/10.2174/1381612811319110006

- Palella, F. J, Jr., Delaney, K.M., Moorman, A. C., Loveless, M. O., Fuhrer, J., Satten G. A.,... Holmberg, S. D. (1998). Declining morbidity and mortality among patients with advanced human immunodeficiency virus infection. HIV outpatient study investigators. The New England Journal of Medicine, 338, 853-860. https:// doi.org/10.1056/NEJM199803263381301.

Perazella, M. A. (2010). Tenofovir-induced kidney disease: an acquired renal tubular mitochondriopathy. Kidney International, 78, 1060-1063. https://doi.org/10.1038/ki.2010.344

- $\quad$ Rotruck, J. T., Rope, A. L., Ganther, H. F., \& Swason, A. B. (1973). Selenium: biochemical role as a component of glutathione peroxidase. Science, 179, 588-90. https://doi.org/10.1126/science.179.4073.588

Sedlak, J., \& Lindsay, R. H. (1968). Estimation of Total, ProteinBound, and Nonprotein Sulfhydryl Groups in Tissue with Ellman's Reagent. Analytical Biochemistry, 25, 1192-1205. https://doi. org/10.1016/0003-2697(68)90092-4

- Shoba, G., Joy, D., Joseph, T., Majeed, M., Rajendran, R., \& Srinivas, P. S. (1998). Influence of piperine on the pharmacokinetics of curcumin in animals and human volunteers. Planta Medica, 64, 353-356. https://doi.org/10.1055/s-2006-957450.

- Sun, M., \& Zigman, S. (1978). An ImprovedSpectrophotometer Assay of Superoxide Dismutase Based On Epinephrine, Antioxidation. Analytical Biochemistry, 90, 81-89. https://doi. org/10.1016/0003-2697(78)90010-6

World Health Organization (WHO) guidelines approved by the guidelines review committee, in consolidated guidelines on the use of antiretroviral drugs for treating and preventing HIV infection: recommendations for a public health approach. World Health Organization: Geneva. 2013. 